\title{
Exploitation of symmetry in periodic Self-Consistent-Field $a b$ initio calculations: application to large three-dimensional compounds
}

\author{
Marco De La Pierre, ${ }^{1,2}$ Roberto Orlando, ${ }^{1}$ Matteo Ferrabone, ${ }^{1}$ \\ Claudio M. Zicovich-Wilson, ${ }^{3}$ and Roberto Dovesi ${ }^{1}$ \\ ${ }^{1}$ Dipartimento di Chimica, Università di Torino and NIS, \\ Nanostructured Interfaces and Surfaces, \\ Centre of Excellence, Via P. Giuria 7, 10125 Torino, Italy \\ ${ }^{2}$ Nanochemistry Research Institute, Department of Chemistry, \\ Curtin University, GPO Box U1987, Perth WA 6845, Australia \\ ${ }^{3}$ Facultad de Ciencias, Universidad Autónoma del Estado de Morelos, \\ Av. Universidad, 1001, Col. Chamilpa, 62209 Cuernavaca (Morelos), Mexico
}

(Dated: June 18, 2014)

\begin{abstract}
Symmetry can dramatically reduce the computational cost (running time and memory allocation) of SelfConsistent-Field $a b$ initio calculations for crystalline systems. Crucial for running time is use of symmetry in the evaluation of one- and two-electron integrals, diagonalization of the Fock matrix at selected points in reciprocal space, reconstruction of the density matrix. As regards memory allocation, full square matrices (overlap, Fock and density) in the Atomic Orbital (AO) basis are avoided and a direct transformation from the packed AO to the SACO (Symmetry Adapted Crystalline Orbital) basis is performed, so that the largest matrix to be handled has the size of the largest sub-block in the latter basis. We here illustrate the effectiveness of this scheme, following recent advancements in the CRYSTAL code, concerning memory allocation and direct basis set transformation. Quantitative examples are given for large unit cell systems, such as zeolites (all-silica faujasite and silicalite MFI) and garnets (pyrope). It is shown that the full SCF of 3D systems containing up to 576 atoms and 11136 Atomic Orbitals in the cell can be run with a hybrid functional on a single core PC with 500 MB RAM in about 8 hours.

Keywords: point symmetry, Symmetry Adapted Crystalline Orbitals, Fock matrix, density matrix, CPU time, memory allocation, quantum-mechanical calculations, CRYSTAL code
\end{abstract}




\section{INTRODUCTION}

When performing electronic structure calculations on crystalline compounds, it is mandatory to exploit translational symmetry by means of a variational basis of Bloch Functions (BFs), so that a block-diagonal structure is obtained for the (infinite) Hamiltonian matrix, each block corresponding to a $\mathbf{k}$ point in the First Brillouin Zone (BZ) (first basis transformation in Figure 1). Actually, all periodic codes are based on the use of BFs. On the other hand, point symmetry is totally or partially neglected in many periodic codes. Whereas most molecular systems containing a large number of atoms have usually low symmetry, a wide range of solids of scientific and/or technological interest have crystalline structures characterized by a relatively large number (usually 6 to 48 ) of symmetry operators, which makes it odd the non-exploitation of symmetry in periodic codes.

In the present paper we discuss how symmetry permits a drastic reduction in running time and memory allocation in Self-Consistent Field (SCF) calculations of the electronic structure for both solids and molecules. This formal scheme as a whole is implemented in the 2014 release of the CRYSTAL code $\mathrm{e}^{1,2}$.

Symmetry can reduce running time at various steps of the SCF process:

1. Calculation of a subset of one- and two-electron integrals; ${ }^{3}$

2. Numerical integration of the exchange-correlation density functional in an asymmetric subset of grid points; ${ }^{3}$

3. Selection of a subset of $\mathbf{k}$ points of the BZ, namely the Irreducible Brillouin Zone (IBZ), at which the Fock matrix is diagonalized; ${ }^{3}$

4. Diagonalization of the Fock matrix by using Symmetry Adapted Crystalline Orbitals $(\mathrm{SACOs}){ }^{4,5}$

5. Reconstruction of the density matrix in the SACO basis.

In order to take full advantage from the description of the wavefunction in the SACO basis, a set of back and forth transformations are required from the Atomic Orbitals (AO) basis. Moreover, representation of the Fock, overlap and density matrices as full square matrices in the AO basis needs always to be avoided. These expedients have been fully implemented for the present study. In the following, performance in terms of both computational time and memory requirements, resulting from full symmetry exploitation, will be illustrated in the case of high symmetry, large 
unit cell three-dimensional compounds: pyrope, all-silica faujasite and silicalite MFI. The maximum number of symmetry operators in crystalline solids is 48 ; another paper has just been submitted, aiming to document the effectiveness of the scheme when the number of operators is larger, as in fullerene molecules and infinite mono-dimensional nanotubes ${ }^{6}$.

The structure of the paper is as follows; in Section II the computational scheme is shown, and the use of symmetry for reducing both the CPU cost and the memory allocation discussed. Section III provides details of the computational setup adopted for the test calculations. The effectiveness of the scheme is documented in Section IV. The main conclusions are drawn in Section V.

\section{THE COMPUTATIONAL SCHEME}

The following scheme has been implemented in the new CRYSTAL14 code. ${ }^{1,2}$ All the presented features are in the current public version, except for the formulation of density matrix in terms of SACOs (point G. below), that is implemented in a second release of the code to be distributed in 2014. A brief outline was presented about two years ago, when the scheme was still at the project stage. ${ }^{7}$

CRYSTAL was designed so as to take advantage of the chemical nature and connectivity of the system. Thus, interactions are computed in real space and selected on the basis of AO overlap criteria. However, as recalled above, the SCF problem for periodic systems must be solved in the reciprocal space by representing matrices in the BF basis. For these reasons, all three types of matrices used in the SCF cycle, namely overlap $(\mathbf{S})$, Fock $(\mathbf{F})$ and density $(\mathbf{P})$, must be represented both in the $\mathrm{AO}$ and the $\mathrm{BF}$ basis along the calculation. Moreover, they all can be stored either in their symmetry irreducible form or in the symmetry full form. In real space, every matrix $\mathbf{M}$ ( $\mathbf{M}$ $=\mathbf{S}, \mathbf{F}, \mathbf{P})$ is expressed in the $\mathrm{AO}$ basis and stored in packed arrays as a result of the application of effective integral selection (screening) criteria, that drastically reduce the number of matrix elements to be computed and stored (see point C. below). Hence their size scales linearly with the size of the system, instead of quadratically. In the following, such matrices are labeled with Latin characters: upper-case for full matrices $\left(\mathrm{M}^{\mathrm{g}}\right)$ and lower-case for matrix irreducible blocks $\left(\mathbf{m}^{\mathbf{g}}\right)$, with $\mathbf{g}$ denoting a real space lattice basis vector. $\mathbf{M}^{\mathrm{g}}$ is obtained from $\mathbf{m}^{\mathbf{g}}$ by application of the symmetry operators $\mathbf{R}$ of the point group. In highly symmetric cases, $\mathbf{m}^{\mathbf{g}}$ is much smaller than $\mathbf{M}^{\mathbf{g}}$ : Table I shows that the size of $\mathbf{F}^{\mathbf{g}}$ and $\mathbf{f}^{\mathbf{g}}$ for all-silica faujasite (primitive cell, labelled FAUp), with accurate basis set and computational conditions (see Section III), is 736056 and 16747, respectively. Their ratio ( $\mathcal{R}_{1}$ in the Table) is 44 , close to the number of symmetry operators, i.e. 
48.

In reciprocal space, $\mathbf{F}$ and $\mathbf{S}$ must both be in square form (this is a capital difference with respect to the direct space representation) to be diagonalized at each $\mathbf{k}$ point in the IBZ and yield the one-electron eigenvalues and eigenvectors. The eigenvectors are also to be stored to memory as they are needed in the reconstruction of the density matrix. They are arranged in a matrix, $\mathbf{A}$, with the same size and symmetry properties related to $\mathbf{F}$. In the following, matrices in reciprocal space (including $\mathbf{A}$ ) are denoted as $\mathbf{M}^{\mathbf{k}}$ when the BFs are constructed in the basis of AOs, whereas blackboard characters $\left(\mathbb{M}^{\mathbf{k}}\right)$ are used when the BFs are in the basis of SACOs so that the overlap, Fock and eigenvectors matrices take a block-diagonal form, each block being labeled by an index of irreducible representation, IR ( $\left.\mathbb{M}^{\mathbf{k}, I R}\right)$.

The reader can appreciate the advantage of using the SACO basis by taking, again, faujasite as an example (Table I): the size of $\mathbf{F}^{\mathbf{k}}$ is $2784 \times 2784$ whereas that of the largest $\mathbb{F}^{\mathbf{k}, I R}$ is only $187 \times 187$ at the $\Gamma$ point, that is, the size of the largest IR matrix to diagonalize and store to memory is about 225 times smaller than $\mathbf{M}^{\mathbf{k}}$ (see $\left(\mathcal{R}_{2}\right)^{2}$ in the Table); other $\mathbf{k}$ points have inevitably smaller symmetry, so that the largest IR matrix is $464 \times 464$, with a saving factor still as large as 36 .

Here follows a list of the steps where CRYSTAL widely benefits from deep use of the system symmetry:

A. Symmetry is used to identify relationships between atoms, shells, atom pairs, so that linear transformations along the SCF process can be performed efficiently through mapping tables. It is also used to find the IRs and the character table, as well as the transformation matrices $\mathbf{W}^{\mathbf{k}}$ from the AO to the SACO basis, which are stored in a compressed form.

B. One- and two-electron integrals are evaluated with reference to the symmetry irreducible set of atoms and shell-shell pairs.

C. Every element of the direct space irreducible Fock matrix wedge $\mathbf{f}^{\mathbf{g}}$ is formed by combination of one- and two-electron integrals with the density matrix $\mathbf{P}^{\mathbf{g}^{\prime}}$ :

$$
\mathbf{f}_{\mu \nu}^{\mathbf{g}}=\sum_{\lambda, \rho, \mathbf{g}^{\prime}} \mathbf{P}_{\lambda \rho}^{\mathbf{g}^{\prime}} \sum_{\mathbf{h}}\left[\left(\mu^{\mathbf{0}} \nu^{\mathbf{g}} \mid \lambda^{\mathbf{h}} \rho^{\mathbf{h}+\mathbf{g}^{\prime}}\right)-\frac{1}{2}\left(\mu^{\mathbf{0}} \lambda^{\mathbf{h}} \mid \nu^{\mathbf{g}} \rho^{\mathbf{h}+\mathbf{g}^{\prime}}\right)\right]+H_{\mu \nu}^{\mathbf{g}}
$$

where square brackets contain the Coulomb and exchange integrals and $H_{\mu \nu}^{\mathrm{g}}$ is the one-electron contribution to the Fock matrix. Summations in Eq. 1 are truncated by screening techniques based on overlap criteria between gaussian functions or charge distributions for multipolar expansions and on an estimate of the density matrix range in real space (see Refs. 1,2,8). In 
the same way, the number of matrix elements to be computed is restricted to those atoms related by some degree of vicinity so that matrices in real space take a very compact structure scaling linearly with the system size, as anticipated above. In addition to this, only the irreducible wedges of the Fock $\left(\mathbf{f}^{\mathrm{g}}\right)$ and density $\left(\mathbf{p}^{\mathbf{g}}\right)$ matrices are formed and stored to memory. Shell-shell blocks of $\mathbf{P}^{\mathrm{g}}$ in Eq. 1 are obtained by rotation from $\mathbf{p}^{\mathbf{g}}$ when needed and this can be done with a "low-memory" approach illustrated in Refs. 9,10. For example, in the case of faujasite (Table I), only 16747 elements of $\mathbf{f}^{\mathbf{g}}$ were computed and stored to memory instead of 736056 in the full matrix $\mathbf{F}^{\mathbf{g}}$, that is, 44 times less elements $\left(\mathcal{R}_{1}\right.$ in the Table).

D. The exchange-correlation functional of the electron density in the unit cell is integrated numerically on a set of points belonging to the asymmetric part of the integration grid.

E. Transformation of the overlap and Fock matrices from real to reciprocal space $\left(\mathbf{m}^{\mathbf{g}} \rightarrow \mathbb{M}^{\mathbf{k}}\right)$ requires in principle the following intermediate transformations:

$$
\mathbf{m}^{\mathrm{g}} \stackrel{a}{\rightarrow} \mathbf{M}^{\mathrm{g}} \stackrel{b}{\rightarrow} \mathbf{M}^{\mathbf{k}} \stackrel{c}{\rightarrow} \mathbb{M}^{\mathbf{k}}
$$

corresponding to:

a) Generation of the full overlap and Fock matrices in real space by applying the set of symmetry operators $\mathbf{R}$ of the point group to the corresponding irreducible matrices:

$$
\mathbf{M}^{\mathbf{g}^{\prime}}=\sum_{\mathbf{R}} \mathbf{R}^{\dagger} \mathbf{m}^{\mathrm{g}} \mathbf{R}
$$

b) Fourier transform of $\mathrm{M}^{\mathrm{g}}$ to reciprocal space, that is, from the $\mathrm{AO}$ to the $\mathrm{BF}$ basis (first transformation in Figure 1):

$$
\mathbf{M}^{\mathbf{k}}=\sum_{\mathbf{g}} e^{i \mathbf{k} \cdot \mathbf{g}} \mathbf{M}^{\mathbf{g}}
$$

c) Transformation of $\mathbf{M}^{\mathbf{k}}$ into the SACO block-diagonal form (second transformation in Figure 1):

$$
\mathbb{M}^{\mathbf{k}}=\mathbf{W}^{\mathbf{k} \dagger} \mathbf{M}^{\mathrm{k}} \mathbf{W}^{\mathbf{k}}
$$

$\mathbf{W}$ matrices take a different form for the various $\mathbf{k}$ points, depending on their multiplicity.

In CRYSTAL09 the three steps in Eq. 2 implied the calculation of the four full matrices at every $\mathbf{k}$ point along subsequent steps. However, since steps $a, b$ and $c$ are mere linear transformations, by an appropriate mapping we can obtain every block of $\mathbb{M}^{\mathbf{k}}\left(\mathbb{M}^{\mathbf{k}, I R}\right)$ in a single step from $\mathbf{m}^{\mathbf{g}}$, 
so reducing dramatically the amount of data to keep in memory with respect to storage of the full square matrices in the AO basis $\left(\mathbf{M}^{\mathbf{k}}\right)$. The required size is equal to the size of the largest matrix $\mathbb{M}^{\mathbf{k}, \mathrm{IR}}\left(M_{I R}\right.$ in Table I). This latter procedure is followed in CRYSTAL14. As already mentioned, in the case of faujasite this amounts to $M_{I R}^{2}=215296$ elements, instead of $N_{A O}^{2}=7750656$ in the AO basis, the ratio between the two allocations, $\left(\mathcal{R}_{2}\right)^{2}$, being about 36 .

F. Every block of the overlap or Fock matrix in the SACO basis $\left(\mathbb{M}^{\mathbf{k}, I R}\right)$ is diagonalized and the eigenvectors $\mathbb{A}^{\mathbf{k}, \mathrm{IR}}$ are obtained. As anticipated, the corresponding saving factor in computing time can be huge.

G. Formation of the irreducible density matrix in real space $\left(\mathbf{p}^{\mathbf{g}}\right)$ was achieved through the following steps in CRYSTAL09:

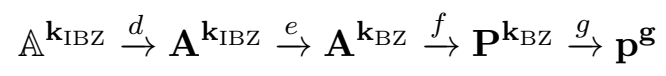

where:

d) The eigenvectors of the Fock matrix in the BF basis for every $\mathbf{k}$ point in the asymmetric part of the first Brillouin zone (IBZ) were obtained from those in the SACO basis. They formed large square matrices with the same size as $\mathbf{F}^{\mathbf{k}}$;

e) The eigenvectors at every $\mathbf{k}$ point in the first Brillouin zone (BZ) were obtained from those in the IBZ by application of all symmetry operators $\mathbf{R}$, because back-Fourier transform to real space requires integration over the entire first Brillouin zone, i.e. a sum over all $\mathbf{k}$ points in the BZ;

f) Formation of the full square density matrix in reciprocal space; ${ }^{8}$

g) Fourier transform of $\mathbf{P}^{\mathbf{k}_{\mathrm{BZ}}}$ to real space.

Here $\mathbf{p}^{\mathrm{g}}$ is in compact form because it takes advantage of both symmetry and interaction screening. In the second release of CRYSTAL14 (to be distributed in 2014), this matrix is generated straightforwardly from the eigenvectors in the SACO basis, $\mathbb{A}^{\mathbf{k}}$, by means of the same mapping that was used for transforming the overlap and Fock matrices from real to reciprocal space (point E. above). Such an improvement has a strong impact both on computational time and memory usage, because full square matrices, such as $\mathbf{A}^{\mathbf{k}}$ and $\mathbf{P}^{\mathbf{k}}$, are no longer to be allocated (their size would be $2784 \times 2784$ in the case of faujasite in Table I).

The new scheme implies the following steps:

$$
\mathbb{A}^{\mathbf{k}_{\mathrm{IBZ}} \stackrel{h}{\rightarrow}} \mathbb{P}^{\mathbf{k}_{\mathrm{IBZ}} \stackrel{i}{\rightarrow}} \mathbb{P}^{\mathbf{k}_{\mathrm{BZ}}} \stackrel{l}{\rightarrow} \mathbf{p}^{\mathbf{g}}
$$


namely:

h) Construction of the density matrix in the SACO basis at reciprocal space points in the IBZ. Unlike step $f$ above, eigenvectors have the size of the corresponding row of the IR they belong to, instead of the full number of the AOs in the unit cell;

i) Generation of the density matrix at all reciprocal space points in the first Brillouin zone from those in the IBZ by application of all symmetry operators (again, arrays are much shorter than in the AO basis adopted in step $e$ above);

l) Fourier transform and basis change from SACOs to AOs. Since such a transformation implies matrix double products, it scales with the third power of the number of basis functions, this being, however, the number of SACOs, which can be much smaller than for the AOs (also in this case the longest $\mathbb{P}^{\mathbf{k}, I R}$ in faujasite contains $M_{I R}=464$ components to be compared with $\left.N_{A O}=2784\right)$.

Note that some of the calculations performed for this paper made use of the parallelized version of CRYSTAL, which builds up on the MPI protocol (www.mpi-forum.org). Two schemes for parallel processing are currently implemented in the code: a replicated data algorithm (PCRYSTAL) ${ }^{9}$, wherein each matrix block as a whole is assigned to a given core, and a distributed data algorithm for massive parallelism (MPPCRYSTAL) ${ }^{10}$, in which large matrix blocks are partitioned and distributed among the cores. The PCRYSTAL version was adopted in this study, which is more suitable for high-symmetry compounds, and for which a new task distribution strategy for the diagonalization step, based on IRs rather than on $\mathbf{k}$ points, has been implemented in the CRYSTAL14 release.

\section{COMPUTATIONAL CONDITIONS}

Simulations were performed with the B3LYP hybrid functional. ${ }^{11-13}$ All atoms were described using a triple zeta basis set with one set of $d$ polarization functions. The level of accuracy in evaluating the two-electron Coulomb and Hartree-Fock exchange series is controlled by five parameters $T_{i}(i=1, \ldots 5) .{ }^{1} T_{1}$ and $T_{2}$ refer to the Coulomb integrals, $T_{3}, T_{4}$ and $T_{5}$ to exchange. In this study accurate values such as $7,7,7,8,18$ were chosen. The DFT exchange-correlation contribution is evaluated by numerical integration over the unit cell volume. In CRYSTAL, radial and angular points of the grid are generated through Gauss-Legendre radial quadrature and Lebedev two-dimensional angular point distributions. A $(75,974)$ p grid was used, corresponding to a pruned grid with 75 radial and 974 angular points (XLGRID keyword in the CRYSTAL14 manual). ${ }^{1}$ The 
reciprocal space was sampled along the lattice vectors according to a sublattice with shrinking factor $^{1}$ set to 2 , corresponding to $3,3,4$ and 8 independent $\mathbf{k}$ vectors in the irreducible part of the Brillouin zone for pyrope, all-silica faujasite with a primitive or a crystallographic cell and silicalite MFI, respectively. Such a choice ensures convergence of the total energy to $10^{-7} \mathrm{Ha} /$ atom. All calculations were run on a cluster with 8 Intel Xeon E5620 cores working at $2.40 \mathrm{GHz}$, and $2 \mathrm{~GB}$ RAM per core.

\section{PERFORMANCE DATA}

We chose three test compounds to document the effectiveness of the presented scheme for SCF calculations: pyrope (PYR), all-silica faujasite (FAUp) and silicalite MFI (MFI). Unit cells are represented in Figure 2; relevant data are shown in Table I. The first two are good examples of solids with highest point group symmetry (48 operators) and large unit cells (80 and 144 atoms, respectively, described here by 1488 and 2784 atomic orbitals). MFI has lower symmetry (8 operators) but twice as many atoms as FAUp (288), as well as 5568 atomic functions in the basis set used. In order to investigate a case with even a larger number of atoms, we performed a second set of calculations on all-silica faujasite using the crystallographic cell as a reference (FAUc), featuring four times the atoms of the primitive cell (576 atoms, with as many as 11136 atomic functions) and still a quite large number of symmetry operators, namely 24 .

Upon application of screening techniques to reduce the number of interactions to be considered, the size of the Fock matrix in real space $\left(S_{\mathbf{F}}\right.$ in Table I) spans between 725000 and 2944224 for the four systems, corresponding to 6 and $22 \mathrm{MB}$ memory occupancy (double precision real numbers). When reduced by symmetry, the size of this matrix $\left(S_{\mathbf{f}}\right.$ in Table I) drops into the range 16634 to 215483 , i.e. $130 \mathrm{~KB}$ to $2 \mathrm{MB}$. The corresponding saving factor $\mathcal{R}_{1}$ is nearly equal to the number of symmetry operators in all cases: 44 for PYR and FAUp, 8 for MFI, 23 for FAUc. Beside accounting for the reduced memory storage with respect to a calculation without symmetry, $\mathcal{R}_{1}$ is also a good estimate of the saving factor in the calculation of one- and two-electron integrals.

Square-shaped matrices can be avoided in almost all steps of the calculation, with the only exception of diagonalization. In this respect, symmetry has dramatic effects, since it permits to perform this step in the basis of SACOs rather than AOs so as to achieve block-decomposition of each $\mathbf{M}^{\mathbf{k}}$ matrix into $N_{I R}$ independent blocks, $\mathbb{M}^{\mathbf{k}, I R}$, as schematically represented in Figure 1. Key quantities are the maximum IR sub-block size $M_{I R}$ and the ratio $\mathcal{R}_{2}=N_{A O} / M_{I R}$, both shown in Table I for the $\Gamma$ point and for the remaining $\mathbf{k}$ points. There are, thus, several relevant 
advantages in such a basis transformation:

i) Memory requirement is dramatically reduced since $M_{I R}^{2}$ is the maximum number of matrix elements to be kept in memory, instead of $N_{A O}^{2}$, if all matrix diagonal blocks are stored to disc and retrieved one by one on need. Saving in memory usage is in the order of $\left(\mathcal{R}_{2}\right)^{2}$;

ii) Diagonalization of many small matrices is to be preferred to that of a larger matrix as the algorithms employed scale with the third power of the matrix size. Here the saving factor is in the order of $\left(\mathcal{R}_{2}\right)^{3} / N_{I R}$;

iii) Only one block of each IR needs to be diagonalized because of degeneracy; eigenvectors for all other rows of an IR are obtained by rotation.

Of course, it must be taken into account that different $\mathbf{k}$ points possess different multiplicity and symmetry properties, such as for example $N_{I R}$. The $\Gamma$ point reflects the full symmetry of the system and exhibits the largest value of $N_{I R}$. On the other hand, the overall performance of the calculation steps involving IRs is inevitably biased by the lowest symmetry $\mathbf{k}$ points, whose IRs have larger size. At the $\Gamma$ point, the largest block in the SACO basis, $M_{I R}$, is about $1 / 16$ of the number of atomic functions $N_{A O}$ for PYR and FAUp, and 1/8 for MFI and FAUc. As a consequence, the memory requirement is $68 \mathrm{~KB}$, instead of $17 \mathrm{MB}$, in the case of PYR; on the other side, it is $16 \mathrm{MB}$ instead of $950 \mathrm{MB}$ for FAUc. As the algorithm for diagonalization has roughly a third-power scaling, at $\Gamma$ the speed-up estimated as $\left(\mathcal{R}_{2}\right)^{3} / N_{I R}$ is about 400 for PYR and 64 for MFI. As already mentioned above, $\mathbf{k}$ points other than $\Gamma$ have always lower symmetry, thus larger IR blocks. Table I shows that value for $\mathcal{R}_{2}$ out of $\Gamma$ is 4 for PYR and FAUc (to be compared with 16 and 8 at $\Gamma$ ), 6 for FAUp and 2 for MFI (compare the two latter with 15 and 8, respectively). In the worst case (among the ones presented here) of MFI, $\mathcal{R}_{2}$ is 2 , which corresponds to a reduction in memory allocation from 240 to $60 \mathrm{MB}$, and an expected speed-up factor of 8 . The real, average speed-up factor over all $\mathbf{k}$ points for a given system will be in between the two extreme situations discussed here, namely $\Gamma$ with high symmetry and a point with low symmetry and large IRs.

Let us now analyze performance and memory requirements into more details. We will consider the steps of an SCF-plus-energy gradient calculation and refer to absolute wall-clock running time instead of purely relative data about scalability, to show data substantiating the performance of the presented scheme when applied to large unit cell compounds under accurate computational conditions, even with low-level hardware. Calculations were run both in serial (single core) and in 
parallel ( 8 cores), so as to show the advantage of the new parallelization strategy of CRYSTAL14 compared to CRYSTAL09: from the IBZ $\mathbf{k}$ points to a finer task farming over the IRs.

Data are reported along Tables II-VI. The various steps of a SCF+gradient calculation were illustrated in Section II. In particular, initialize includes steps preliminary to the SCF procedure, among which the construction of tables for screening and symmetry analysis. orthog is also preliminary to the SCF procedure and refers to the orthogonalization of the AO basis set. In a direct-SCF strategy every SCF cycle is made up of the evaluation of the integrals (integrals), numerical integration of the exchange-correlation density functional $(d f t)$, transformation from $\mathbf{f}^{\mathbf{g}}$ to $\mathbb{F}^{\mathbf{k}}$ in block-diagonal form (Fock), diagonalization of every $\mathbb{F}^{\mathbf{k}, I R}$ block (diag), construction of the density matrix and its transformation to $\mathbf{p}^{\mathbf{g}}$ (densmat). TOT $T_{c y c}$ refers to a complete SCF cycle. The four systems considered in the present study converged to an accuracy of $10^{-8} \mathrm{Ha}$ in the total energy in about $20 \mathrm{SCF}$ cycles. The total time required to complete a SCF calculation is labeled as $T O T_{S C F}$ in the Tables. Data about the calculation of the total energy gradient with respect to nuclear positions are also reported $(\mathrm{grad})$ as being an important step in structure optimizations and calculation of several other properties such as phonons, elastic tensors etc.

Table II shows running times on a single core for the various steps of the SCF+gradient calculation. In all cases the most time-consuming portion is the calculation of integrals, accounting for $60 \div 80 \%$ of the time required for a single SCF cycle. Integration of the exchange-correlation functional (step $d f t$ ) is either the second (PYR, FAUp) or third (MFI, FAUc) most expensive step. MFI and FAUc have larger number of atoms (thus of atomic orbitals) and lower symmetry compared to PYR and FAUp. Beside that, diagonalization grows with the third power of the sizes of matrices to diagonalize. As a consequence this step accounts for about $1 \%$ the cycle time for PYR and FAUp, whereas it grows to $25 \%$ for MFI and FAUc. Notably, transformation from AO to SACO basis set (step Fock) takes a negligible amount of time in all cases; reconstruction of the density matrix (densmat step) accounts for no more than 3\%. Preliminary steps to the SCF calculation, namely classifications and symmetry analysis (initialize) and basis set orthogonalization (orthog), require more time for the two systems having larger size, MFI and FAUc. However, considering that they need to be performed only once per simulation, in all cases their cost turns out to be negligible with respect to the whole SCF procedure.

In order to explicitly show how beneficial the use of symmetry is in the SCF scheme, in Table III we present the running times obtained when symmetry is not considered. Values in parentheses are the ratios with respect to the corresponding data in Table II discussed above. As expected, in the case of the calculation of the integrals, this ratio is very close to $\mathcal{R}_{1}$ (Table I): 48, 52, 9 and 26 
are to be compared with 44,44 and 8 and 23 . The initialization step scales with about the same ratio. Timings corresponding to transformation from AO to SACO basis must tend to zero when symmetry is not exploited; as a consequence, the corresponding ratios are not significant. The diagonalization step is worth being discussed into some detail. The corresponding saving ratios are about 170, 200, 13 and 110 for PYR, FAUp, MFI and FAUc. However, as anticipated at the beginning of this Section, these global values sort out as an average among all the $\mathbf{k}$ points. Let us then discuss a specific case, namely that of the $\Gamma$ point, whose timings are singled out in row diag $\Gamma$ in Tables II and III. The corresponding saving ratios are 330, 290, 26 and 170, obviously larger than the global ones, as $\Gamma$ shows the highest symmetry in the reciprocal space. These four values are of the same order of magnitude than the ones obtained using the approximated scaling rule $\left(\mathcal{R}_{2}\right)^{3} / N_{I R}$ : 410, 330, 60 and 92. Finally, considering the overall SCF time, we notice its saving ratio is as large as 42, 43, 10 and 44 for PYR, FAUp, MFI and FAUc, being essentially related to the integrals and diag steps. The former dominates the cycle time of PYR and FAUp, whereas both are relevant in the case of MFI and FAUc. For all systems a smaller influence is also due to the $d f t$ step.

Assessing the scaling of the algorithm for three-dimensional compounds is not easy, as a series of compounds with homogeneously increasing size is not available. The best analysis we can perform is considering unit cells of different size for the same compound, even if point symmetry can change, thus partially biasing comparison. Here we compare the primitive and conventional cells of all-silica faujasite (see Table II), which differ by a factor of 4 in the number of atoms and by $1 / 2$ in the number of symmetry operators. The total time required for a SCF calculation grows from 2500 to 28000 seconds, i.e. about 11 times, to be compared with an ideal factor of 8 for linear scaling, as there are four times as many atoms and half the symmetry operators in FAUc with respect to FAUp. In particular, the integrals, dft, Fock and initialize steps scale by a factor of about 10, densmat of 20, whereas orthog and diag show a by far larger increase, around 100, as the time required for the diagonalization step scales roughly with $M_{I R}^{3}$, and $M_{I R}$ increases by a factor 6 .

Table IV shows running times in the case of parallel calculations exploiting 8 cores. Total SCF time reduces by a factor around 7 for PYR and FAUp, and 5 for MFI and FAUc, with efficiencies of about 90 and $60 \%$, respectively. This good performance is driven by the nearly ideal scaling of the calculation of integrals and integration of the exchange-correlation functional. As regards MFI and FAUc, the main step affecting efficiency is diagonalization; the reason is essentially task unbalancing among the various cores, due to the very different sizes of the various $\mathbb{F}^{\mathbf{k}, \text { IR }}$, so that diagonalization of matrices with size $M_{I R}$ becomes dominant. Step densmat scales in a similar 
way for the same reason.

Peak memory usage is reported in Table V. The maximum amount of required memory along all the calculation is about 100 (PYR), 110 (FAUp), 410 (MFI) and 520 (FAUc) MB. The most memory consuming step is the reconstruction of the density matrix in all cases but MFI, where it is overtaken by the Fock and diag steps. Table VI shows memory use when symmetry exploitation is switched off. Maximum allocation along the entire SCF cycle increases by a factor of about 3 , 5, 4 and 9 in PYR, FAUp, MFI and FAUc, respectively, reaching values of about 270, 530, 1500 and $4900 \mathrm{MB}$. Memory saving achieved with symmetry is satisfactory, but compared to the case of computational time the corresponding saving factors are smaller. In fact, a relevant amount of memory is employed to store information that is independent of the use of symmetry.

\section{CONCLUSIONS}

We have presented a scheme for the Self-Consistent-Field calculations of periodic systems through full exploitation of both translational and point symmetry.

Symmetry can largely reduce running time and memory requirements during integrals calculation, integration of exchange-correlation functional, sampling of the reciprocal space, diagonalization of the Fock matrix, reconstruction of the density matrix. Specific expedients have been implemented for this publication, namely direct transformation from AO to SACO basis and avoidance of allocation of full square matrices.

The efficiency of the scheme has been documented in the case of large unit cell, highly symmetric compounds, such as pyrope, all-silica faujasite and silicalite MFI. Computational time and memory allocation have been discussed for the various steps of the SCF calculation. Calculation of integrals and integration of the exchange-correlation functional are the most time-consuming steps; the cost of diagonalizing the Fock matrix rapidly increases with the size of the system. Saving time with respect to a brute force calculation with no symmetry is roughly in the order of the number of symmetry operators. Scaling with system size, estimated comparing calculations on primitive and conventional cells of the same compound, is closer to a linear rather than quadratic behaviour. When performing parallel calculations, efficiency is around $60 \div 90 \%$ using 8 computing cores; the main bottle-neck is represented by the diagonalization step, due to the very different sizes of the IR sub-blocks which reduces task balancing among cores.

As a synthetic figure, it turns out that a full SCF calculation on the conventional cell of faujasite, with 576 atoms and 11136 atomic functions, takes as little as 8 hours on a machine with one core, 
requiring only $500 \mathrm{MB}$ of memory.

\section{ACKNOWLEDGEMENTS}

The authors acknowledge Compagnia di San Paolo for financial support (Progetti di Ricerca di Ateneo-Compagnia di San Paolo-2011-Linea 1A, progetto ORTO11RRT5). CZ acknowledges financial support from Mexican CONACyT through project CB-178853.

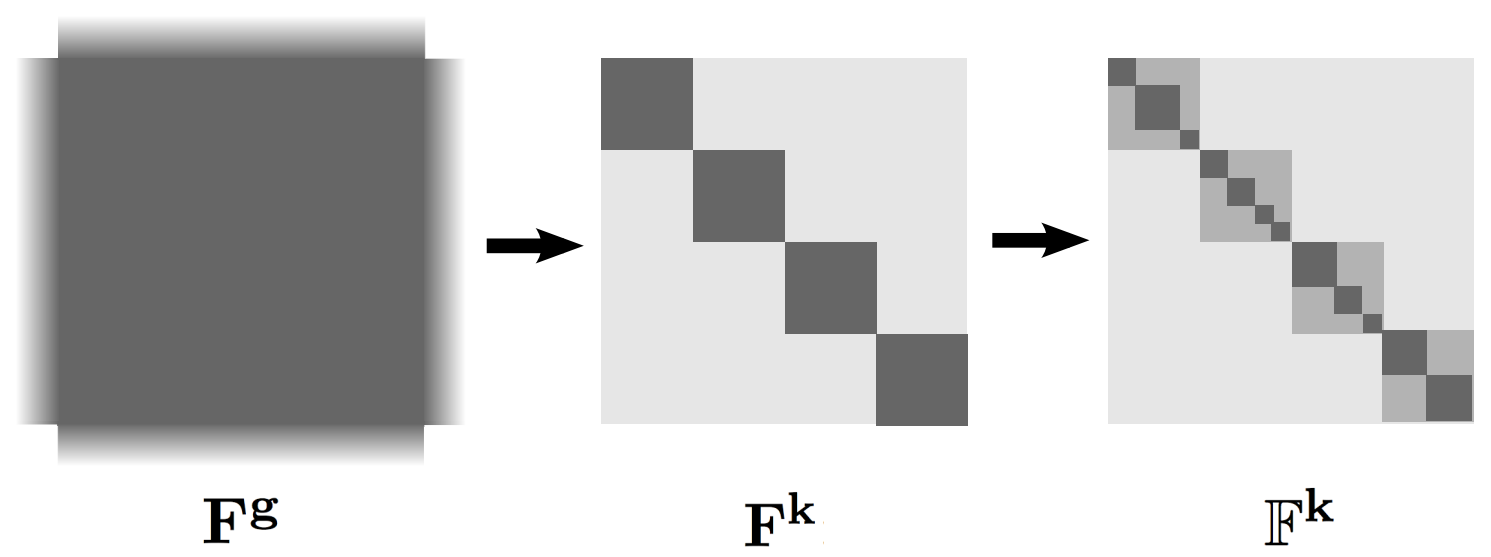

FIG. 1: Block-factorization of the Fock matrix in periodic SCF calculations. $\mathbf{F}^{\mathbf{g}}$ : basis of AOs (Atomic Orbitals, non-packed form; borders are blurry to indicate that such matrix is infinite in principle); $\mathbf{F}^{\mathbf{k}}$ : basis of BFs (Bloch Functions); $\mathbb{F}^{\mathbf{k}}$ : basis of SACOs (Symmetry Adapted Crystalline Orbitals). 

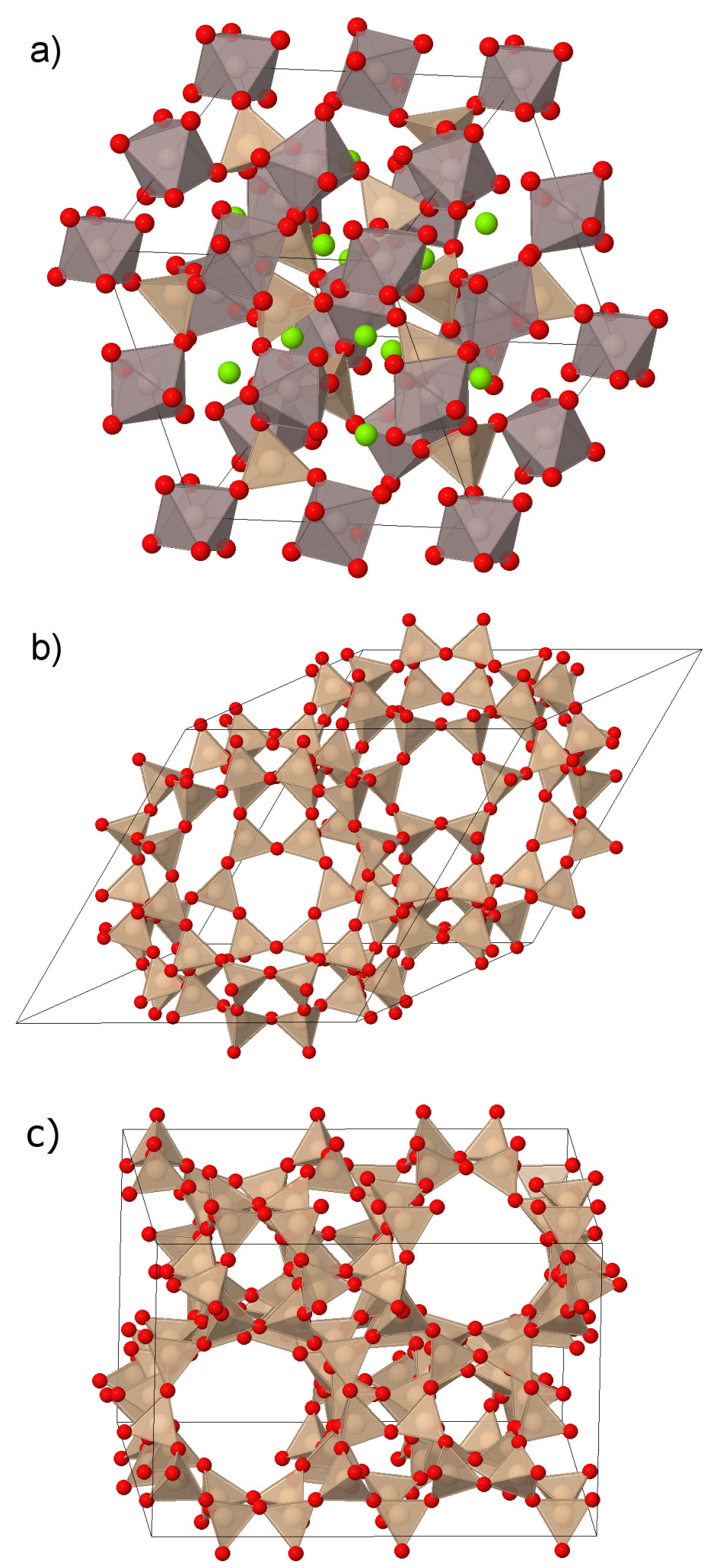

FIG. 2: Graphical representation of the primitive unit cells of a) pyrope, b) all-silica faujasite, and c) silicalite MFI. Some atoms have been added, that belong to neighboring cells, in order to show complete coordination polyhedra. 


\begin{tabular}{|c|c|c|c|c|c|c|c|c|c|c|c|c|c|c|}
\hline & $N_{o p}$ & $N_{a t}^{a s y m}$ & $N_{a t}^{t o t}$ & $N_{A O}$ & $N_{\mathrm{k}}$ & $\begin{array}{c}{ }^{N} \\
\Gamma\end{array}$ & $\begin{array}{l}J_{I R} \\
\text { Oth. }\end{array}$ & $S_{\mathrm{fg}}$ & $S_{\text {Fg }}$ & $\begin{array}{c}M \\
\Gamma\end{array}$ & $\begin{array}{l}I_{I R} \\
\text { Oth. }\end{array}$ & $\mathcal{R}_{1}$ & & $\begin{array}{l}\mathcal{R}_{2} \\
\text { Oth. }\end{array}$ \\
\hline PYR & 48 & 4 & 80 & 1488 & 3 & 10 & $2 ; 3$ & 16634 & $725^{\prime} 000$ & 93 & 374 & 44 & 16 & 4 \\
\hline FAUp & 48 & 5 & 144 & 2784 & 3 & 10 & $4 ; 6$ & 16747 & $736 ’ 056$ & 187 & 464 & 44 & 15 & 6 \\
\hline MFI & 8 & 38 & 288 & 5568 & 8 & 8 & $1 ; 2 ; 4$ & 215483 & $1^{\prime} 704^{\prime} 216$ & 712 & 2784 & 8 & 8 & 2 \\
\hline FAUc & 24 & 30 & 576 & 11136 & 4 & 5 & 5 & 127740 & 2'944'224 & 1440 & 2784 & 23 & 8 & 4 \\
\hline
\end{tabular}

TABLE I: Effect of symmetry on SCF calculations for a series of high-symmetry solids: pyrope (PYR), silicalite MFI (MFI), primitive (p) and conventional (c) cells of all-silica faujasite (FAU). $N_{o p}$ is the number of symmetry operators; $N_{a t}^{a s y m}, N_{a t}^{t o t}$ and $N_{A O}$ are the number of irreducible atoms, the total number of atoms and the total number of AOs in the basis set, respectively; $N_{\mathbf{k}}$ and $N_{I R}$ are the number of irreducible k points and of IRs, respectively. $S_{\mathbf{f g}}$ and $S_{\mathbf{F g}}$ are the size of the irreducible and reducible (both packed) Fock matrices in direct space; $M_{I R}$ is the maximum IR sub-block size. $\mathcal{R}_{1}$ and $\mathcal{R}_{2}$ are the ratios $S_{F} / S_{f}$ and $N_{A O} / M_{I R}$, respectively. Values for $N_{I R}, M_{I R}$ and $\mathcal{R}_{2}$ are given separately for $\Gamma$ and other $\mathbf{k}$ points. Regarding $N_{I R}$ for $\mathbf{k}$ points other than $\Gamma$, for FAUc all 3 points have a value of 5 , while for MFI there are 2 points with 1, 4 points with 2 , and 1 point with 4 . 


\begin{tabular}{l|c|c|c|c}
\hline & PYR & FAUp & MFI & FAUc \\
\hline initialize & 9.36 & 6.84 & 123.12 & 73.40 \\
orthog & 0.24 & 0.63 & 41.88 & 43.22 \\
integrals & 170.44 & 75.62 & 1397.64 & 821.77 \\
dft & 41.58 & 42.91 & 271.92 & 223.15 \\
Fock & 0.12 & 0.22 & 0.95 & 1.60 \\
diag & 0.68 & 2.60 & 647.92 & 315.19 \\
diag $\Gamma$ & 0.02 & 0.16 & 16.04 & 19.51 \\
densmat & 0.91 & 1.72 & 28.69 & 35.78 \\
TOT $_{\text {cyc }}$ & 213.77 & 123.10 & 2347.15 & 1397.53 \\
TOT & \\
SCF & 4285.00 & 2469.47 & 47108.00 & 28067.22 \\
gradient & 1351.74 & 650.41 & 10620.98 & 5885.82 \\
\hline
\end{tabular}

TABLE II: Running time (seconds) of the SCF+gradient calculations with one single core. Data refer to the following steps: 1) initialization of the simulation, including the construction of the symmetry group and transformation matrices (initialize); 2) orthogonalization of the basis set (orthog); 3) calculation of one- and two-electron integrals (integrals); 4) numerical integration of the exchange-correlation density functional $(d f t) ; 5)$ transformation of $\mathbf{f}^{\mathbf{g}}$ into $\mathbb{F}^{\mathbf{k}}$ (Fock); 6) Fock matrix block-diagonalization (diag); 7) Contribution to diag for the $\Gamma$ point only $(\operatorname{diag} \Gamma) ; 8)$ construction of the density matrix (densmat); 9) a single SCF cycle (TOT $T_{c y c}$, corresponding to a step 3-8 sequence); 10) the entire SCF procedure $\left(T O T_{S C F}, 20 \mathrm{SCF}\right.$ cycles at the chosen conditions); 11) calculation of the total energy gradients with respect to the nuclear positions (gradient). 


\begin{tabular}{|c|c|c|c|c|c|c|c|c|}
\hline \multirow[b]{2}{*}{ initialize } & \multicolumn{2}{|c|}{ PYR } & \multicolumn{2}{|c|}{ FAUp } & \multicolumn{2}{|c|}{ MFI } & \multicolumn{2}{|c|}{ FAUc } \\
\hline & 427.05 & $(45.6)$ & 292.69 & $(42.8)$ & 1039.78 & (8.4) & 1686.64 & $(23.0)$ \\
\hline orthog & 15.07 & $(62.8)$ & 99.06 & $(157.2)$ & 718.07 & (17.1) & 5796.12 & (134.1) \\
\hline integrals & 8103.48 & $(47.5)$ & 3912.40 & $(51.7)$ & 12035.45 & $(8.6)$ & 21156.91 & $(25.7)$ \\
\hline$d f t$ & 712.62 & (17.1) & 828.14 & (19.3) & 1842.86 & (6.8) & 4067.00 & $(18.2)$ \\
\hline Fock & 0.17 & (1.4) & 0.55 & $(2.5)$ & 2.30 & $(2.4)$ & 12.99 & (8.1) \\
\hline diag & 116.38 & (171.1) & 507.82 & $(195.2)$ & 8240.35 & $(12.7)$ & 35876.57 & (113.8) \\
\hline $\operatorname{diag} \Gamma$ & 7.93 & $(330.5)$ & 46.78 & $(292.4)$ & 416.15 & $(25.9)$ & 3241.32 & (166.1) \\
\hline densmat & 6.89 & $(7.6)$ & 24.48 & $(14.2)$ & 126.32 & $(4.4)$ & 437.94 & $(12.2)$ \\
\hline$T O T_{c y c}$ & 8939.57 & (41.8) & 5273.42 & $(42.8)$ & 22247.34 & $(9.5)$ & 61551.54 & $(44.0)$ \\
\hline$T O T_{S C F}$ & 179233.52 & $(41.8)$ & 105860.15 & $(42.9)$ & 446704.65 & $(9.5)$ & 1238513.56 & $(44.1)$ \\
\hline gradient & 56383.43 & (41.7) & 27347.14 & $(42.0)$ & 84248.80 & (7.9) & 134572.70 & $(22.9)$ \\
\hline
\end{tabular}

TABLE III: Running time (seconds) of the SCF+gradient calculations with one single core, when symmetry is not exploited; see caption to Table II as a legend. Data in parentheses are the ratios between these times and the ones obtained using symmetry (Table II).

\begin{tabular}{l|cc|cc|cc|cc}
\hline & \multicolumn{2}{|c|}{ PYR } & \multicolumn{2}{c|}{ FAUp } & \multicolumn{2}{c|}{ MFI } & \multicolumn{2}{c}{ FAUc } \\
\hline initialize & 5.72 & $(20)$ & 2.62 & $(33)$ & 18.81 & $(82)$ & 14.62 & $(63)$ \\
orthog & 0.09 & $(33)$ & 0.28 & $(28)$ & 20.01 & $(26)$ & 19.87 & $(27)$ \\
integrals & 24.03 & $(89)$ & 10.37 & $(91)$ & 179.85 & $(97)$ & 107.49 & $(96)$ \\
dft & 5.41 & $(96)$ & 5.57 & $(96)$ & 36.31 & $(94)$ & 29.93 & $(93)$ \\
Fock & 0.03 & $(47)$ & 0.06 & $(49)$ & 0.20 & $(60)$ & 0.33 & $(61)$ \\
diag & 0.31 & $(28)$ & 0.77 & $(42)$ & 251.29 & $(32)$ & 124.74 & $(32)$ \\
densmat & 0.39 & $(29)$ & 0.66 & $(33)$ & 9.72 & $(37)$ & 13.31 & $(34)$ \\
TOT $_{\text {cyc }}$ & 30.16 & $(89)$ & 17.40 & $(88)$ & 477.39 & $(61)$ & 275.67 & $(63)$ \\
TOT $_{S C F}$ & 609.01 & $(88)$ & 350.90 & $(88)$ & 9586.62 & $(61)$ & 5547.89 & $(63)$ \\
gradient & 189.43 & $(89)$ & 87.40 & $(93)$ & 1376.14 & $(96)$ & 772.70 & $(95)$ \\
\hline
\end{tabular}

TABLE IV: Running time (seconds) of the SCF+gradient calculations when 8 core are used; see caption to Table II as a legend. Data in parentheses represent the percent efficiency of the scaling, taking the single core calculation as a reference (Table II). 


\begin{tabular}{l|c|c|c|c}
\hline & PYR & FAUp & MFI & FAUc \\
\hline initialize & 55 & 73 & 215 & 233 \\
orthog & 57 & 76 & 287 & 331 \\
integrals & 67 & 85 & 202 & 251 \\
dft & 64 & 80 & 182 & 221 \\
Fock + diag & 60 & 79 & 411 & 452 \\
densmat & 97 & 110 & 355 & 524 \\
TOT & \\
SCF & 97 & 110 & 411 & 524 \\
gradient & 197 & 213 & 390 & 478 \\
\hline
\end{tabular}

TABLE V: Memory peaks (MBytes) of the SCF+gradient calculations with one single core; see caption to Table II as a legend.

\begin{tabular}{l|c|c|cc|cc}
\hline & PYR & FAUp & \multicolumn{2}{|c|}{ MFI } & \multicolumn{2}{|c}{ FAUc } \\
\hline initialize & $266(4.8)$ & $494(6.8)$ & $956(4.5)$ & 1900 & $(8.2)$ \\
orthog & $190(3.3)$ & $398(5.3)$ & $1008(3.5)$ & 2935 & $(8.9)$ \\
integrals & $201(3.0)$ & $321(3.8)$ & 630 & $(3.1)$ & 1190 & $(4.7)$ \\
dft & $192(3.0)$ & $310(3.9)$ & 607 & $(3.3)$ & $1153(5.2)$ \\
Fock+diag & $243(4.1)$ & $533(6.7)$ & $1523(3.7)$ & $4893(10.8)$ \\
densmat & $271(2.8)$ & $512(4.6)$ & $1384(3.9)$ & $4098(7.8)$ \\
TOT $T_{S C F}$ & $271(2.8)$ & $533(4.8)$ & $1523(3.7)$ & $4893(9.3)$ \\
gradient & $311(1.6)$ & $467(2.2)$ & $1316(3.4)$ & $4008(8.4)$ \\
\hline
\end{tabular}

TABLE VI: Memory peaks (MBytes) of the SCF+gradient calculations with one single core, when symmetry is not exploited; see caption to Table II as a legend. Data in parentheses are the ratios between these allocations and the ones obtained using symmetry (Table V). 
1 Dovesi R, Saunders VR, Roetti C, Orlando R, Zicovich-Wilson CM, Pascale F, Civalleri B, Doll K, Harrison NM, Bush IJ, D’Arco Ph, Llunell M, Causà M, Noël Y. CRYSTAL14 User's Manual. Università di Torino, Torino, 2014

2 Dovesi R, Orlando R, Erba A, Zicovich-Wilson CM, Civalleri B, Casassa S, Maschio L, Ferrabone M, De La Pierre M, D'Arco Ph, Noël Y, Causà M, Rérat M, Kirtman B. CRYSTAL14: A Program for the Ab Initio Investigation of Crystalline Solids. Int. J. Quantum Chem., 2014, DOI:10.1002/qua.24658

3 Dovesi R. On the Role of Symmetry in the Ab Initio Hartree-Fock Linear-Combination-of-AtomicOrbitals Treatment of Periodic Systems. Int. J. Quantum Chem., 1986, 29:1755-1774

4 Zicovich-Wilson CM, Dovesi R. On the Use of Symmetry-Adapted Crystalline Orbitals in SCF-LCAO Periodic Calculations. I. The Construction of the Symmetrized Orbitals. Int. J. Quantum Chem., 1998, 67:299-309

5 Zicovich-Wilson CM, Dovesi R. On the Use of Symmetry-Adapted Crystalline Orbitals in SCF-LCAO Periodic Calculations. II. Implementation of the Self-Consistent-Field Scheme and Examples. Int. J. Quantum Chem., 1998, 67:311-320

6 Orlando R, De La Pierre M, Zicovich-Wilson CM, Erba A, Dovesi R. On the full exploitation of symmetry in periodic (as well as molecular) Self-Consistent-Field ab initio calculations. Submitted to J. Chem. Phys., 2014.

7 Zicovich-Wilson CM, Noël Y, Ferrari AM, Orlando R, De La Pierre M, Dovesi R. On the use of symmetry in SCF calculations. The case of fullerenes and nanotubes. AIP Conf. Proc., 2012, 1456:248-255

8 Pisani C, Dovesi R, Roetti C. Hartree-Fock ab-initio of crystalline systems, Volume 48 of Lect. N. Chem. Heidelberg: Spinger Verlag, 1988.

9 Bush IJ, Tomic S, Searle BG, Mallia G, Bailey CL, Montanari B, Bernasconi L, Carr JM, Harrison NM. Parallel implementation of the ab initio CRYSTAL program: electronic structure calculations for periodic systems. Proc R. Soc. A, 2011, 467:2112-2126

10 Orlando R, Delle Piane M, Bush IJ, Ugliengo P, Ferrabone M, Dovesi R. A New Massively Parallel Version of CRYSTAL for Large Systems on High Performance Computing Architectures. J. Comput. Chem., 2012, 33:2276-2284

11 Becke AD. Density functional theochemistry. III. The role of exact exchange. J. Chem. Phys., 1993, 98:5648-5652

12 Lee C, Yang W, Parr RG. Development of the Colle-Salvetti correlation-energy formula into a functional of the electron density. Phys. Rev. B, 1988, 37:785-789

13 Stephens PJ, Devlin FJ, Chabalowski CF, Frisch MJ. Ab initio calculation of vibrational absorption and circular dichroism spectra using density functional force fields. J. Phys. Chem., 1994, 98:11623-11627 